\title{
MicroRNA-1271 inhibits proliferation and promotes apoptosis of multiple myeloma cells through inhibiting smoothened-mediated Hedgehog signaling pathway
}

\author{
ZHENGWEI XU ${ }^{1,2}$, CHEN HUANG $^{1}$ and DINGJUN HAO ${ }^{2}$ \\ ${ }^{1}$ Department of Cell Biology and Genetics, Xi'an Jiaotong University Health Science Center, \\ Xi'an, Shaanxi 710061; ${ }^{2}$ Department of Spine Surgery, Xi'an Jiaotong University \\ Medical College Honghui Hospital, Xi'an, Shaanxi 710054, P.R. China
}

Received June 24, 2016; Accepted November 16, 2016

DOI: $10.3892 / o r .2016 .5304$

\begin{abstract}
MicroRNAs (miRNAs) have emerged as important regulators in multiple myeloma (MM). miR-1271 is a tumor suppressor in many cancer types. However, the biological role of miR-1271 in MM remains unclear. In the present study, we elucidated the biological role of miR-1271 in MM. Results showed that miR-1271 was significantly decreased in primary MM cells from MM patients and MM cell lines. Overexpression of miR-1271 inhibited proliferation and promoted apoptosis of MM cells. Conversely, suppression of miR-1271 showed the opposite effect. Bioinformatics algorithm analysis predicted that smoothened (SMO), the activator of Hedgehog $(\mathrm{HH})$ signaling pathway, was a direct target of miR-1271 that was experimentally verified by a Dual-luciferase reporter assay. Furthermore, overexpression of miR-1271 inhibited SMO expression and HH signaling pathway. Conversely, the restoration of SMO expression markedly abolished the effect of miR-1271 overexpression on cell proliferation, apoptosis and HH signaling pathway in MM cells. Taken together, the present study suggests that miR-1271 functions as a tumor suppressor that inhibits proliferation and promotes apoptosis of MM cells through inhibiting SMO-mediated $\mathrm{HH}$ signaling pathway. This finding implies that miR-1271 is a potential therapeutic target for the treatment of MM.
\end{abstract}

Correspondence to: Dr Chen Huang, Department of Cell Biology and Genetics, Xi'an Jiaotong University Health Science Center, No. 76 Yanta West Road, Xi'an, Shaanxi 710061, P.R. China E-mail: huangchenxa@sina.com

Abbreviations: miRNAs, microRNA; MM, multiple myeloma; SMO, smoothened; UTR, untranslated region; HH, Hedgehog; Gli1, glioma-associated oncogene homolog 1

Key words: multiple myeloma, miR-1271, Hedgehog, SMO, cell proliferation, cell apoptosis

\section{Introduction}

Multiple myeloma (MM) is one of the most common hematological cancers characterized by high proliferation of plasma cells expressing the surface marker CD138 in the bone marrow $(1,2)$. Despite the advancement in treatment options over the past decades, MM remains an intractable disease with a poor survival rate and a relatively high incidence rate in recent years $(3,4)$. The underlying mechanism of the pathogenesis of MM remains poorly understood. Therefore, it is of great importance to develop novel and effective therapies for MM.

In recent years, microRNAs (miRNAs) have been taken as promising therapeutic targets for cancer treatments, including MM (5). miRNAs are a type of small, non-coding regulatory RNA molecules (20-25 nucleotides) that post-transcriptionally and negatively modulate target gene expression by targeting the 3'-untranslated region (UTR) of target gene $(6,7)$. Therefore, miRNAs regulate a variety of cellular processes, including cell proliferation, apoptosis, differentiation, migration and invasion (8). Increasing evidence has reported that numerous miRNAs are frequently dysregulated in MM serving as oncogenes or tumor suppressors in regulating MM cell proliferation, apoptosis, cell cycle, drug resistance, migration and invasion (9-15). In addition, the dysregulated miRNAs also represent potential biomarkers for MM diagnosis and prognosis (16-18). However, the precise mechanism of miRNAs in MM remains largely unknown.

Hedgehog (HH) signaling pathway is a highly conserved pathway from Drosophila to vertebrates that regulate embryonic development and adult tissue homeostasis (19). Three HH ligands exist including desert hedgehog, Indian hedgehog and sonic hedgehog in mammals $(20,21)$. In the state of inactivation, the HH receptor patched1 (Ptch1) inhibits smoothened (SMO), which is a seven-transmembrane protein essential for $\mathrm{HH}$ activation (22). When HH ligands bind to Ptch1, SMO is released and activated, which then initiates the transcriptional program of $\mathrm{HH}$ signaling including Ptch1 and gliomaassociated oncogene homolog 1 (Gli1) $(20,23)$. Although $\mathrm{HH}$ signaling pathway is essential for embryonic development, tissue repair, and homeostasis, the abnormal activation of $\mathrm{HH}$ signaling pathway can lead to tumorigenesis $(22,24-27)$. The 
abnormal activation of HH signaling pathway has been found in solid tumors (prostate, pancreatic and lung cancers) (28-30) and hematologic malignancies (MM and B-cell lymphoma) (31-33). HH signaling pathway regulates cell proliferation, apoptosis, survival, drug resistance, colony growth and selfrenewal of MM cells (34-36). Therefore, strategies inhibiting $\mathrm{HH}$ signaling may be a promising and effective anticancer intervention.

A recent study has suggested that miR-1271 functions as a tumor suppressor gene in various cancer types (37-40). However, whether miR-1271 plays a potential role in MM is unknown. In this study, we investigated the potential role and underlying mechanism of miR-1271 in MM. We found that miR-1271 was significantly decreased in MM samples and MM cell lines. Overexpression of miR-1271 inhibited proliferation and promoted apoptosis of MM cells. Bioinformatics algorithms analysis showed that SMO was a predicted target gene of miR-1271 that was confirmed by a Dual-luciferase reporter assay. The overexpression of miR-1271 inhibited SMO expression as well as HH signaling in MM cells. The restoration of SMO expression significantly abrogated the effects of miR-1271. The present study suggests that miR-1271 functions as a tumor suppressor of MM, indicating a potential molecular candidate for MM treatment.

\section{Materials and methods}

Cell cultures. Bone marrow aspirates were collected from 10 MM patients and 10 healthy donors in Xi'an Jiaotong University Medical College Red Cross Hospital. The primary MM cells were isolated from bone marrow aspirates as previously described (41). Briefly, mononuclear cells were separated from bone marrow aspirates by Ficoll-Hypaque density gradient centrifugation (Amersham, Little Chalfont, UK). Then, the cells were suspended in ice-cold phosphate-buffered saline (PBS) and incubated with microbeads labeled with a mouse anti-human CD138 monoclonal antibody (Miltenyi Biotech, Auburn, CA, USA). The CD138 ${ }^{+}$cells were sorted on a BD fluorescence-activated cell sorting FACSAria flow cytometer (BD Biosciences, San Jose, CA, USA) in accordance with the manufacturer's instruction. The collection and use of clinical samples were approved by the Institutional Human Experiment and Ethics Committee of Xi'an Jiaotong University Medical College Red Cross Hospital with written informed consents from all MM patients and healthy donors. The human MM cell lines including NCI-H929, U266 and MM1.R were purchased from the American Type Culture Collection (ATCC; Manassas, VA, USA). The KMS-12-BM MM cell line was purchased from Beijing Abace Biology, Co., Ltd. (Beijing, China). The primary MM cells, human MM cell lines, and normal plasma cells (nPCs) were cultured in RPMI-1640 (Gibco, Rockville, MD, USA) in supplement with $10 \%$ fetal bovine serum ( FBS; Gibco) and 1\% penicillin/streptomycin mix (Sigma-Aldrich, St. Louis, MO, USA) in a humidified atmosphere containing $5 \% \mathrm{CO}_{2}$ at $37^{\circ} \mathrm{C}$.

Real-time quantitative PCR (RT-qPCR) analysis. Total RNA from tissues or cells was extracted using TRIzol reagent (Invitrogen, Carlsbad, CA, USA). Complementary DNA was synthesized using M-MLV reverse transcriptase (BioTeke,
Beijing, China) for mRNA expression analysis or miScript reverse transcription kit (Qiagen, Dusseldorf, Germany) for miRNA expression analysis. PCR amplification was performed on an Applied Biosystems AB 7500 Real-Time PCR system (Applied Biosystems, In., Carlsbad, CA, USA) using Power SYBR-Green PCR Master Mix (Applied Biosystems). The primers used were as follows: miR-1271 forward, 5'-CA GCACTTGGCACCTAGCA-3' and reverse, 5'-TATGGTTGT TCTCCTCTCTGTCTC-3'; U6 forward, 5'-CGCTTCGGC AGCACATATACTAA-3' and reverse, 5'-TATGGAACGC TTCACGAATTTGC-3'; SMO forward, 5'-TGCTCATCGT GGGAGGCTACTT-3' and reverse, 5'-ATCTTGCTGGCAG CCTTCTCAC-3'; Gli1 forward, 5'-TATGGACCTGGCTTT GGA-3' and and reverse, 5'-CCTATGTGAAGCCCTATTT GC-3'; Ptch1, CTCTGGAGCAGATTTCCAAGG; Ptch1 forward, 5'-CTCTGGAGCAGATTTCCAAGG-3' and reverse, 5'-TGCCGCAGTTCTTTTGAATG-3' and GAPDH forward, 5'-CCATGTTCGTCATGGGTGTG-3' and reverse, 5'-GGTG CTAAGCAGTTGGTGGTG-3'. U6 was used as the internal control of miR-1271 and GAPDH was used as the internal control of mRNA. The relative gene expression was determined by $2^{-\Delta \Delta C t}$ method, normalized against GAPDH or U6, and then compared with control.

Cell transfection. The miR-1271 mimics, miR-1271 inhibitor, and their scrambled controls (Scr) were purchased from Shanghai GenePharma (Shanghai, China). Cells were transfected with miR-1271 mimics or miR-1271 inhibitor at a final concentration of $50 \mathrm{nM}$ using Lipofectamine 2000 (Invitrogen). The open reading frame of SMO without 3'-UTR was inserted into pcDNA3.1 (BioVector, Beijing, China). For overexpression of SMO, the pcDNA/SMO constructs were transfected into cells using Lipofectamine 2000 (Invitrogen).

Colony formation assay. Cells were transfected with miR-1271 mimics or miR-1271 inhibitor for $48 \mathrm{~h}$. Then, the cells $(200$ cells/well) were seeded in 6 -well plates and grown in a medium containing $0.3 \%$ noble agar for 2 weeks at $37^{\circ} \mathrm{C}$. After fixing with $100 \%$ methanol for $30 \mathrm{~min}$, the cells were stained with $0.1 \%$ crystal violet (Sigma-Aldrich). The number of colonies was observed and counted under a microscope (Olympus Corp., Tokyo, Japan).

3-(4,5-Dimethyl-thiazol-2-yl)-2,5-diphenyltetrazolium bromide (MTT) assay. Cell proliferation was assessed by MTT assay. Briefly, cells were seeded into a 96-well plate at a density of $5 \times 10^{3}$ cells/well and transfected with miR-1271 mimics or miR-1271 inhibitor for 24, 48 and $72 \mathrm{~h}$. After replacement with fresh medium, $20 \mu 1$ MTT ( $0.5 \mathrm{mg} / \mathrm{ml}$ in PBS; Sigma) was added to each well and incubated for $4 \mathrm{~h}$ at $37^{\circ} \mathrm{C}$. Then, the supernatant was discarded and $200 \mu \mathrm{l}$ dimethyl sulfoxide (Sigma-Aldrich) was added to each well. After incubation for $15 \mathrm{~min}$, the absorbance at $490 \mathrm{~nm}$ was measured with a microplate reader (Thermo Fisher Scientific, Rockford, IL, USA).

Cell apoptosis assay. Cell apoptosis was determined by the terminal deoxynucleotidyl transferase dUTP nick end labeling (TUNEL) and caspase-3 activity assay. For TUNEL assay, briefly, cells were fixed with $4 \%$ paraformaldehyde and permeabilized with $0.2 \%$ Triton X-100 followed by incuba- 
tion with TUNEL reaction mixtures (Roche Diagnostics, Indianapolis, IN, USA) for $1 \mathrm{~h}$ at $37^{\circ} \mathrm{C}$. The apoptotic cells were observed under a microscope (Olympus), and five fields per slide were randomly chosen to quantitatively calculate the apoptotic cells. For caspase-3 activity assay, cells were lysed and the protein concentration was measured. A total of $100 \mu \mathrm{g}$ of protein with $50 \mu \mathrm{l}$ of reaction buffer was treated with $5 \mu \mathrm{l}$ DEVD-pNA substrate ( $4 \mathrm{mM}$; Roche Diagnostics) for $2 \mathrm{~h}$ at $37^{\circ} \mathrm{C}$. The absorbance at $405 \mathrm{~nm}$ was measured with a microplate reader (Thermo Fisher Scientific).

Dual-luciferase reporter assay. The 3'-UTR of SMO containing the target sequence of miR-1271 was inserted into pmirGLO vector (Promega, Madison, WI, USA) to obtain pmirGLO-SMO 3'-UTR, and the 3'-UTR of SMO containing mutant miR-1271 target sites was inserted into pmirGLO vector (Promega) to obtain pmirGLO-mutant SMO 3'-UTR. NCI-H929 cells were co-transfected with pmirGLO-SMO 3'-UTR or pmirGLO-mutant SMO 3'-UTR and miR-1271 mimics, miR-1271 inhibitor or Scr controls. At $4 \mathrm{~h}$ after incubation, the luciferase activity was detected by a Dual-luciferase reporter assay system (Promega).

Western blot analysis. Total proteins were extracted, separated and transferred onto a nitrocellulose membrane (Bio-Rad Laboratories, Hercules, CA, USA). The membrane was blocked by $5 \%$ non-fat milk and incubated with primary antibodies at $4^{\circ} \mathrm{C}$ overnight. After three washes with Tris-buffered saline containing $0.1 \%$ Tween-20, the membrane was incubated with horseradish peroxidase conjugated secondary antibodies (1:2,000; Bosis, Beijing, China) for $1 \mathrm{~h}$ at $37^{\circ} \mathrm{C}$. Then, the protein was detected using enhanced chemiluminescence (Millipore, Boston, MA, USA). The primary antibodies (antiSMO and anti-GAPDH) used in this study were purchased from Santa Cruz Biotechnology (Santa Cruz, CA, USA). The intensities of protein bands were quantified by Image-Pro Plus 6.0 software (Media Cybernetics, Inc., Rockville, MD, USA). The relative protein expression was normalized against GAPDH and then compared with the control.

Data analysis. All the data were reported as means \pm standard deviation. Statistical analyses were analyzed by the Student's t-test for two group comparison or one-way analysis of variance followed by Bonferroni post hoc for multiple group comparison (>2) using SPSS version 11.5 (SPSS, Inc., Chicago, IL, USA). At $\mathrm{P}<0.05$, the difference was considered statistically significant.

\section{Results}

Expression of miR-1271 is downregulated in MM. To investigate whether miR-1271 plays a role in MM, we firstly examined the miR-1271 expression in primary MM cells isolated from MM patient bone marrow samples. The results showed that the expression of miR-1271 was significantly lower in MM primary cells than in plasma cells from healthy donors (Fig. 1A). We then evaluated miR-1271 expression in MM cell lines including NCI-H929, KMS-12-BM, U266 and MM1.R. As compared with normal plasma cells (nPCs), miR-1271 was significantly decreased in MM cell lines
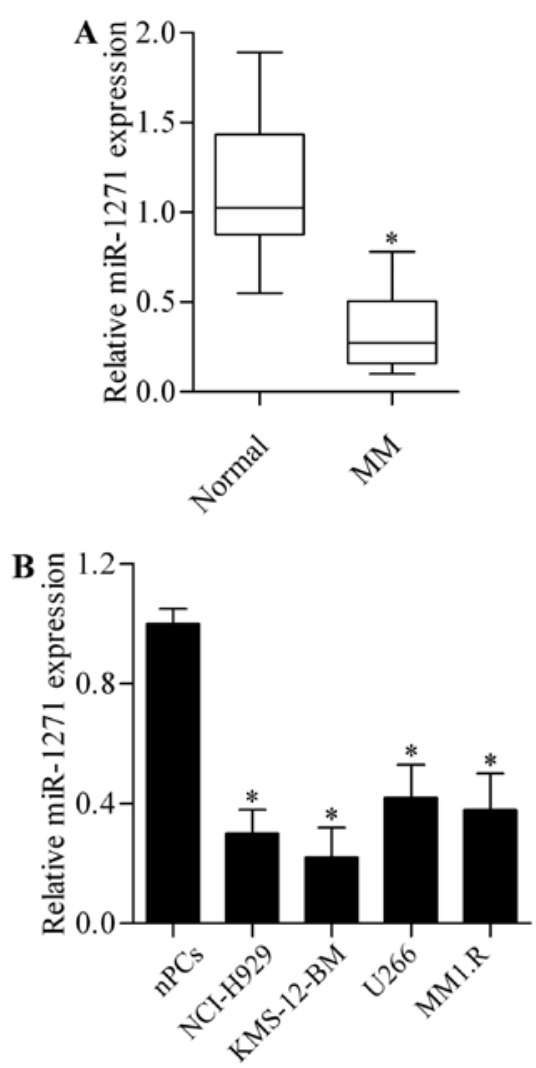

Figure 1. Detection of miR-1271 expression in MM by RT-qPCR (A) Expression of miR-1271 in primary MM cells derived from MM patients. Plasma cells from healthy donors (normal) were used as control. ${ }^{*} \mathrm{P}<0.05$ vs. normal. (B) The expression of miR-1271 in MM cell lines including NCI-H929, KMS-12-BM, U266 and MM1.R compared with normal plasma cells (nPCs). ${ }^{*} \mathrm{P}<0.05$ vs. nPCs.

(Fig. 1B). These results suggest a tumor suppressor role of miR-1271 in MM.

miR-1271 inhibits MM cell proliferation. To explore the biological role of miR-1271 in MM, we performed gainof- and loss-of-function experiments in KMS-12-BM and NCI-H929 cells by transfection of miR-1271 mimics or miR-1271 inhibitor. The results showed that transfection of miR-1271 significantly increased the expression of miR-1271 in KMS-12-BM (Fig. 2A) and NCI-H929 (Fig. 2B) cells, whereas miR-1271 inhibitor markedly decreased miR-1271 expression. We then detected the effect of miR-1271 on cell proliferation by MTT assay. The results showed that overexpression of miR-1271 significantly suppressed MM cell proliferation and miR-1271 inhibition markedly promoted cell proliferation (Fig. 2C and D). Furthermore, the colony growth of KMS-12-BM (Fig. 2E) and NCI-H929 (Fig. 2F) cells was also significantly decreased or increased by miR-1271 overexpression or miR-1271 inhibition, respectively. These data indicate that miR-1271 inhibit MM cell proliferation.

miR-1271 induces MM cell apoptosis. To further investigate the function of miR-1271 in MM, we then evaluated its effect on cell apoptosis. TUNEL assay showed that miR-1271 overexpression markedly induced cell apoptosis of MM cells, whereas miR-1271 inhibition markedly suppressed MM cell 

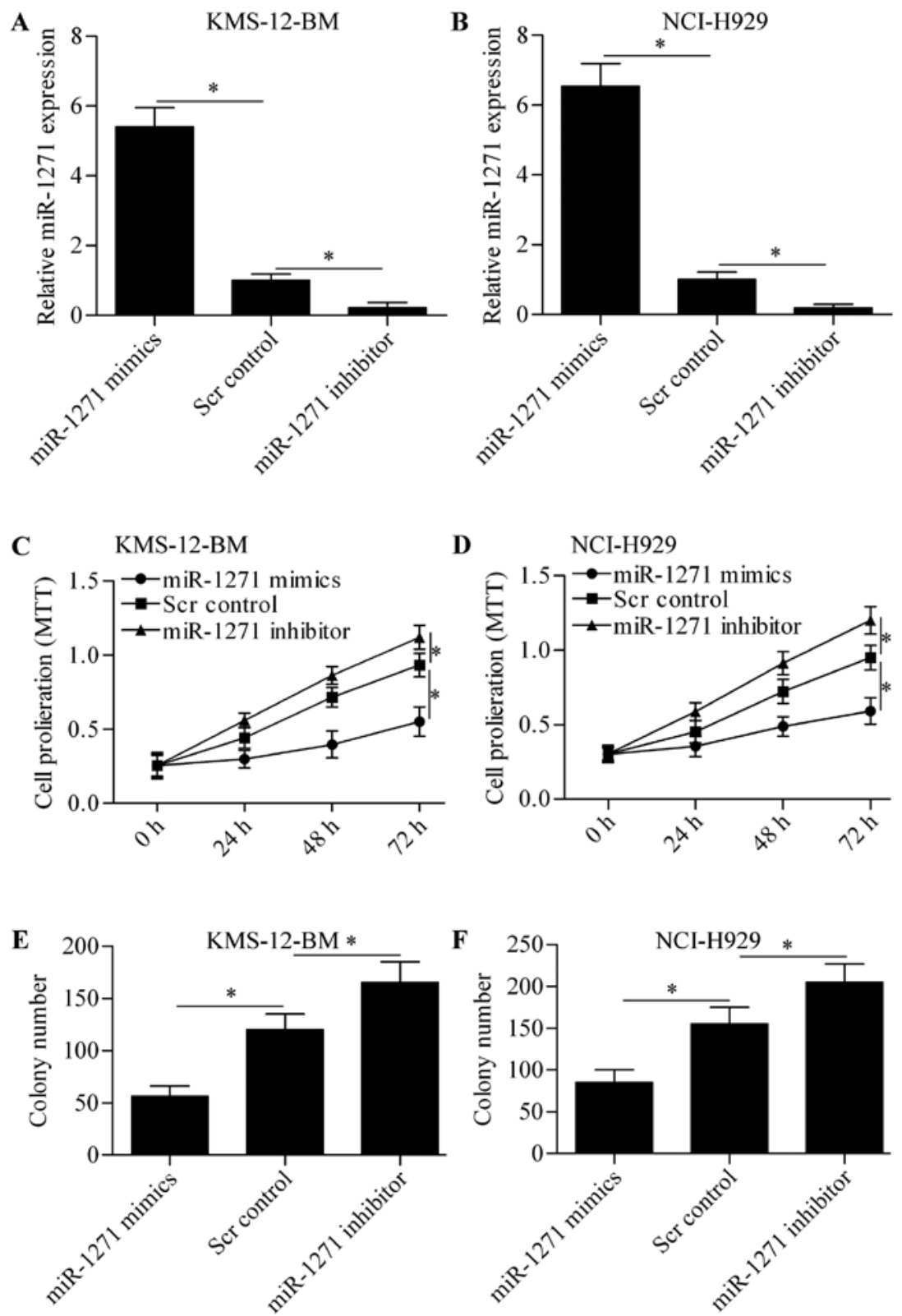

Figure 2. miR-1271 inhibits MM cell proliferation. RT-qPCR analysis of miR-1271 expression in KMS-12-BM (A) and NCI-H929 (B) cells transfected with miR-1271 mimics or miR-1271 inhibitor. Cells transfected with scrambled oligonucleotides (Scr) were used as control. The effect of miR-1271 on cell proliferation of KMS-12-BM (C) and NCI-H929 (D) cells was detected by MTT assay. The effect of miR-1271 on colony forming ability of KMS-12-BM (E) and NCI-H929 (F) cells was detected by colony formation assay. ${ }^{*} \mathrm{P}<0.05$.

A

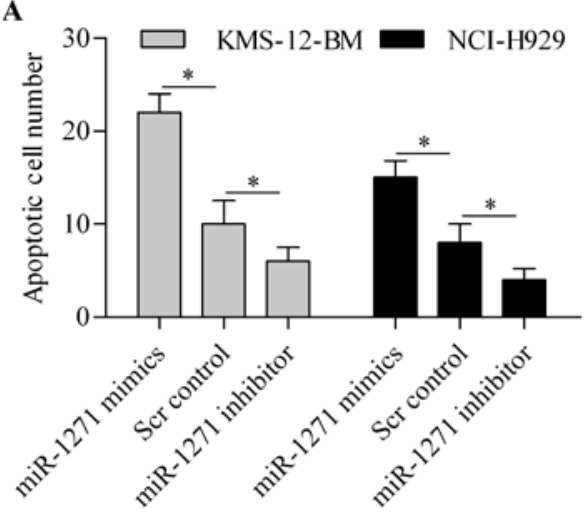

B

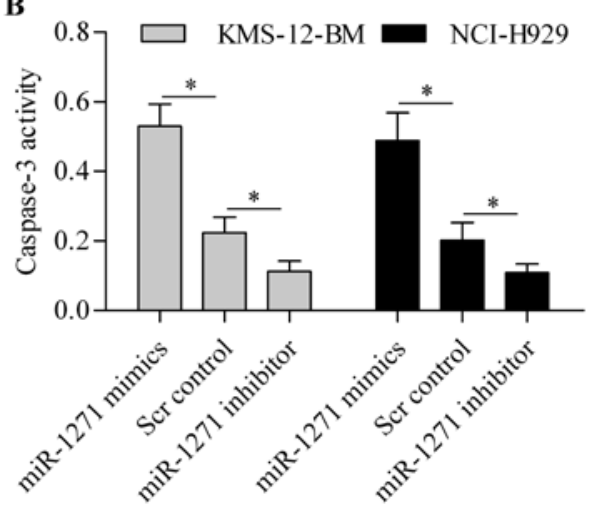

Figure 3. miR-1271 induces MM cell apoptosis. KMS-12-BM or NCI-H929 cells were transfected with miR-1271 mimics or miR-1271 inhibitor and incubated for $48 \mathrm{~h}$. Cell apoptosis was determined by TUNEL assay (A) or (B) caspase- 3 activity assay. ${ }^{*} \mathrm{P}<0.05$. 

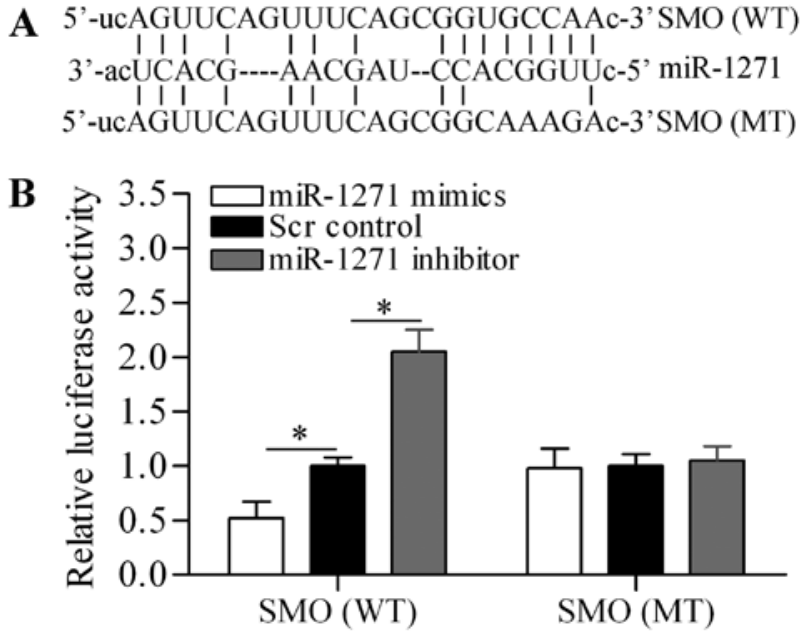

Figure 4. miR-1271 targets the 3'-UTR of SMO. (A) The targeting sites between miR-1271 and the 3'-UTR of SMO. Mutant sequences in the 3'-UTR of SMO are indicated. (B) Relative luciferase activity of NCI-H929 cells cotransfected with WT or MT SMO 3'-UTR luciferase reporter constructs and miR-1271 mimics, miR-1271 inhibitor, or Scr control. * $\mathrm{P}<0.05$

apoptosis (Fig. 3A). Moreover, caspase-3 activity assay showed that the activity of caspase-3 was significantly upregulated by miR-1271 overexpression but decreased by miR-1271 inhibition (Fig. 3B). These results suggest that miR-1271 induces apoptosis of MM cells.

miR-1271 targets the 3'-UTR of SMO. To understand the underlying mechanism by which miR-1271 inhibits proliferation and induces apoptosis of MM cells, we used bioinformatics analysis to seek the potential targets of miR-1271. Notably, we found that SMO, an important oncogene (42), was predicted as a potential target gene of miR-1271 (Fig. 4A). To verify the targeting relationship, luciferase reporter vectors containing wild-type (WT) or mutant (MT) SMO 3'-UTR were constructed. The Dual-luciferase reporter assay showed that miR-1271 mimics significantly decreased the luciferase activity of SMO 3'-UTR (WT), whereas miR-1271 inhibitor significantly increased the luciferase activity of SMO 3'-UTR (WT) (Fig. 4B). However, neither miR-1271 mimics nor miR-1271 inhibitor showed significant effect on the luciferase activity of SMO 3'-UTR (WT). Subsequently, we performed RT-qPCR and western blot analysis to detect the direct effect of miR-1271 on SMO expression. The results showed that expression of both mRNA (Fig. 5A and B) and protein (Fig. 5C and D) was significantly decreased by miR-1271 mimics or increased by miR-1271 inhibitor in MM cells. Taken together, these results indicate that miR-1271 inhibits SMO expression by directly targeting the 3'-UTR of SMO.

miR-1271 inhibits the HH signaling pathway. Because SMO is the critical regulator of $\mathrm{HH}$ signaling pathway, we speculated that miR-1271 might affect the $\mathrm{HH}$ signaling pathway. To test this hypothesis, we detected the effect of miR-1271 on Gli1 and Ptch1 expression. The results showed that the mRNA expression of Gli1 (Fig. 6A) and Ptch1 (Fig. 6B) was significantly downregulated by miR-1271 overexpression. By contrast, suppression of miR-1271 markedly increased the expression of
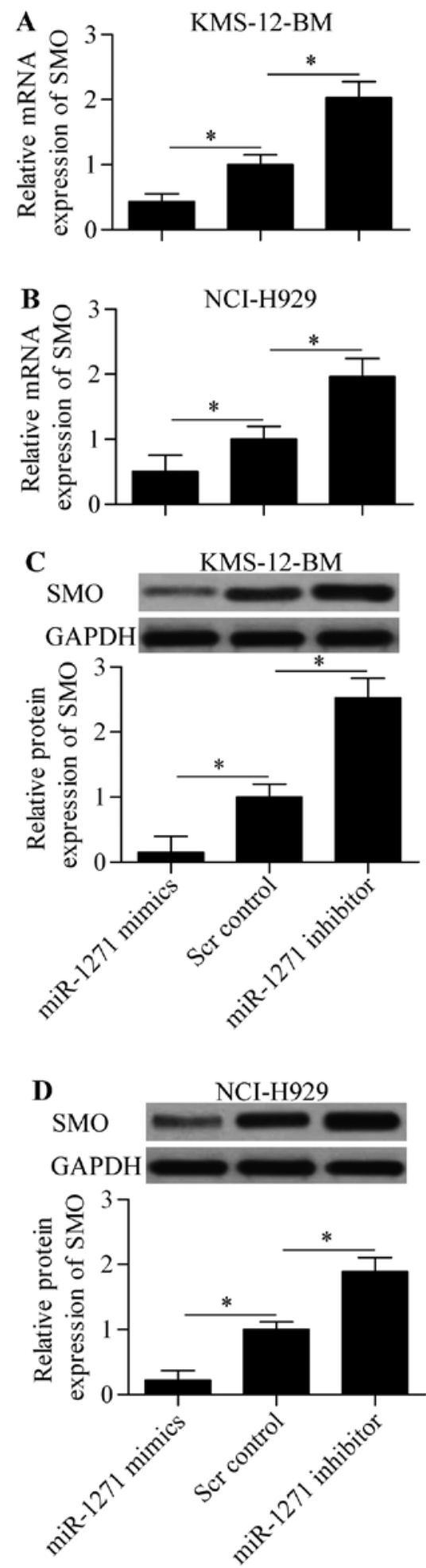

Figure 5. miR-1271 regulates SMO expression. KMS-12-BM or NCI-H929 cells were transfected with miR-1271 mimics and miR-1271 inhibitor for $48 \mathrm{~h}$ and then subjected to analysis. The mRNA expression of SMO in KMS-12-BM (A) and NCI-H929 (B) cells was detected by RT-qPCR analysis. The protein expression of SMO in KMS-12-BM (C) and NCI-H929 (D) cells was detected by western blot analysis. ${ }^{*} \mathrm{P}<0.05$.

Gli1 (Fig. 6A) and Ptch1 (Fig. 6B) in MM cells. These results suggest that miR-1271 regulates the $\mathrm{HH}$ signaling pathway.

Overexpression of SMO reverses the effect of $m i R-1271$. To validate whether miR-1271 functions through SMO, 
A
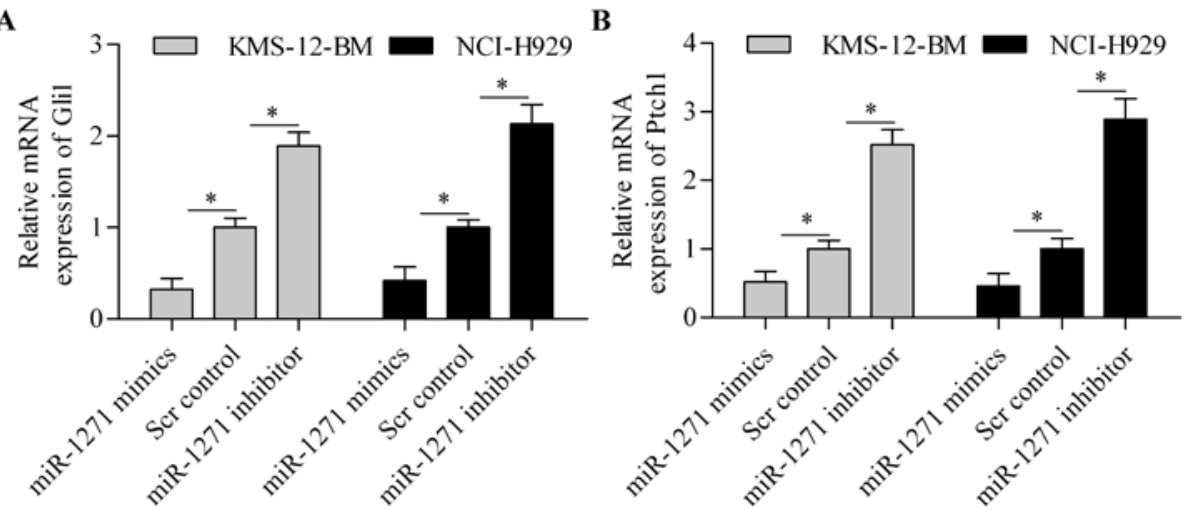

Figure 6. miR-1271 inhibits the HH signaling pathway. RT-qPCR analysis of Gli1 (A) and Ptch1 (B) expression in KMS-12-BM and NCI-H929 cells. KMS-12-BM and NCI-H929 cells were transfected with miR-1271 mimics or miR-1271 inhibitor for $48 \mathrm{~h}$ and then subjected to analysis. " $\mathrm{P}<0.05$.
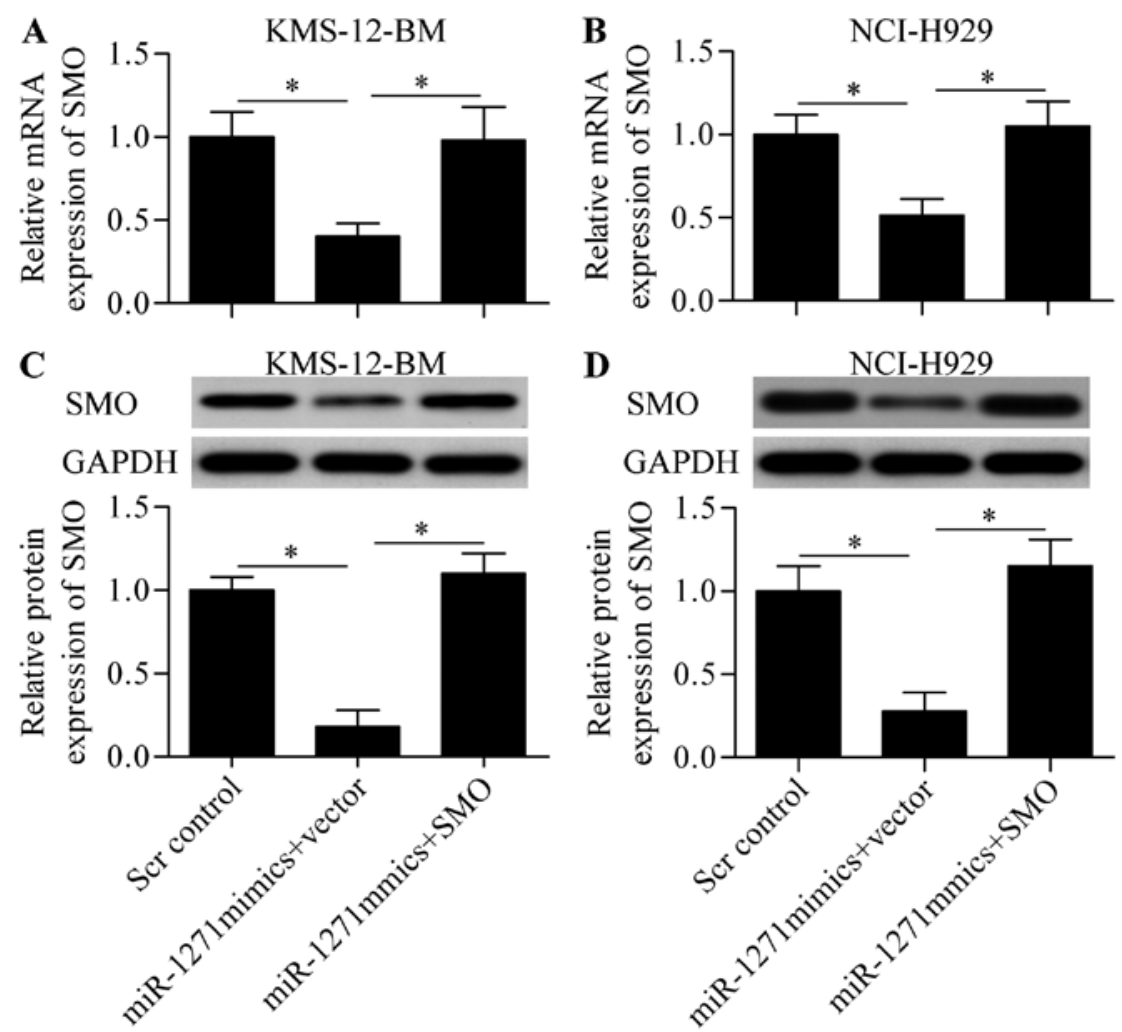

Figure 7. Restoration of SMO expression. Cells were transfected with Scr control, miR-1271 mimics plus null vector, or miR-1271 mimics plus SMOoverexpressing vectors and incubated for $48 \mathrm{~h}$. The mRNA of SMO in KMS-12-BM (A) and NCI-H929 (B) cells was detected by RT-qPCR. The protein expression of SMO in KMS-12-BM (C) and NCI-H929 (D) was detected by western blot analysis. ${ }^{*} \mathrm{P}<0.05$.

we constructed a SMO-overexpressing vector harboring no 3'-UTR of SMO and performed a rescue experiment. The cells were co-transfected with miR-1271 mimics and SMO-overexpressing vector. The results showed that the decreased mRNA (Fig. 7A and B) and protein (Fig. 7C and D) expression induced by miR-1271 was significantly restored by SMO-overexpressing vector transfection. Notably, overexpression of SMO expression significantly restored MM cell proliferation, which was suppressed by miR-1271 (Fig. 8A). Furthermore, the promotion effect of miR-1271 on cell apoptosis was markedly reversed by SMO overexpression (Fig. 8B). In addition, the inhibitory effect of miR-1271 on Gli1 (Fig. 8C) and Ptch1 (Fig. 8D) expression was restored by SMO overex- pression, thereby implying that SMO overexpression reversed the inhibitory effect of miR-1271 on HH signaling pathway.

\section{Discussion}

In the present study, we established a tumor suppressor role of miR-1271 in MM. The expression of miR-1271 was significantly downregulated in MM cells. Importantly, overexpression of miR-1271 inhibited proliferation and promoted apoptosis of MM cells. Further data demonstrated that SMO was a direct target gene of miR-1271 that overexpression of miR-1271 inhibited SMO expression. Suppression of SMO by miR-1271 overexpression also significantly inhibited the $\mathrm{HH}$ 

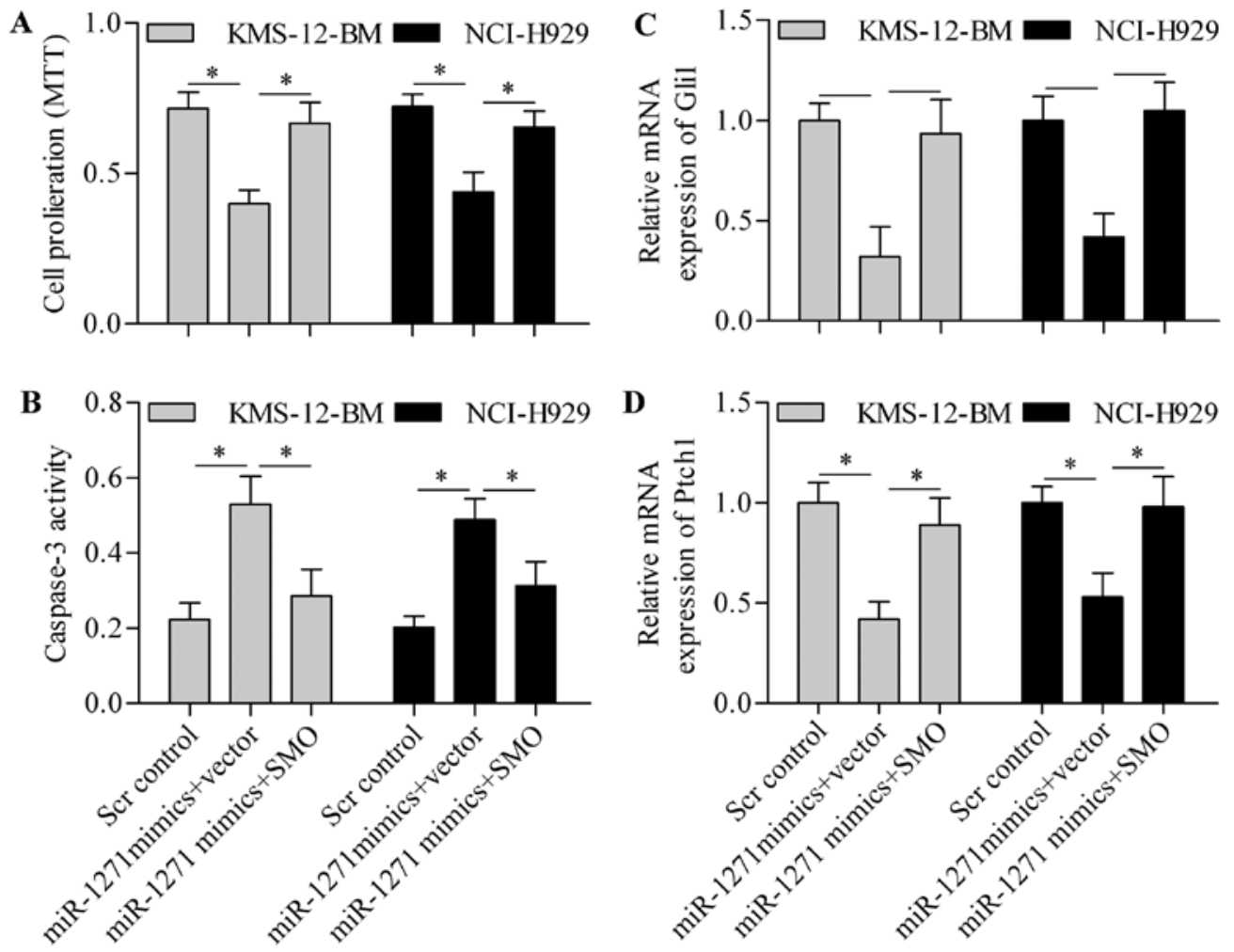

Figure 8. SMO overexpression reverses the effect of miR-1271. Cells were transfected with Scr control, miR-1271 mimics plus null vector, or miR-1271 mimics plus SMO-overexpressing vectors and incubated for $48 \mathrm{~h}$. Cell proliferation (A) and apoptosis (B) were detected by MTT assay and caspase-3 activity, respectively. The expression of Gli1 (C) and Ptch1 (D) was detected by RT-qPCR analysis. "P<0.05.

signaling pathway. However, these effects of miR-1271 overexpression were markedly reversed by SMO overexpression. This study for the first time report the expression and function of miR-1271 in MM.

miRNAs play an important role in tumorigenesis acting as either oncogenes or tumor suppressors, representing an attractive therapeutic target (43). In recent years, miR-1271 has been reported as tumor-associated genes that are involved in various cancer types and functions through different target genes. A functional screening identifies that miR-1271 is a tumor suppressor in hepatocellular carcinoma that is decreased in tumor samples and inhibits tumor cell growth through targeting glypican-3 (44). In gastric cancer, miR-1271 is downregulated and inversely correlated with tumor size, tumor stage and lymph node metastasis (40). Yang et al (45) reported that miR-1271 inhibits gastric cancer cell proliferation and promotes the sensitization to cisplatin-induced apoptosis through targeting insulin-like growth factor 1 receptor, insulin receptor substrate 1, serine/threonine-protein kinase mTOR, and anti-apoptotic protein Bcl-2. miR-1271 is decreased in oral squamous cell carcinoma tissues and cell lines and overexpression of miR-1271 inhibits cell proliferation, colony formation, migration, and invasion of oral squamous cell carcinoma cells through targeting anaplastic lymphoma kinase (46). More recently, Liu et al (37) reported that miR-1271 functioned as tumor suppressor in pancreatic cancer through targeting ZEB1 and TWIST1. Tumor suppressor role of miR-1271 is also found in ovarian (39) and lung cancer (38) through targeting cyclin G1 or mTOR, respectively. Interestingly, an oncogenic role of miR-1271 is also reported that miR-1271 promotes cell proliferation and invasion of non-small cell lung cancer cells through inhibiting homeobox A5 (47). However, in this study, we have demonstrated that miR-1271 is downregulated in MM cells and overexpression of miR-1271 inhibits proliferation and induces apoptosis of MM cells, supporting a tumor suppressor role of miR-1271.

SMO is an activator of the HH signaling pathway that functions as oncogene in various cancers (42). Overexpression of SMO is associated with aberrant activation of $\mathrm{HH}$ signaling pathway (42). Peacock et al (33) reported that HH signaling pathway maintains a tumor stem cell trait of MM cells. In MM cells, inhibition of SMO induces a decrease in cell viability and inhibits the HH signaling pathway (48). Inhibiting $\mathrm{HH}$ signaling pathway inhibits proliferation of MM cells $(34,36)$. A new drug named vismodegib involves the inhibition of HH pathway and shows promising results in the treatment of medulloblastoma and basal-cell carcinoma (49). Therefore, strategies inhibiting $\mathrm{HH}$ signaling represent promising and effective anticancer intervention for MM.

miRNAs have emerged as promising tools for cancer treatment because of the inhibitory effect of miRNAs on gene expression. Indeed, increasing evidence has accumulated on the potential miRNAs that can directly target and inhibit SMO expression and the HH signaling pathway. miR-388-3p has been reported to target SMO to inhibit liver cancer cell invasion (50) and colorectal cancer cell growth and invasion $(51,52)$. In chronic myeloid leukemia cells, inhibition of SMO by miR-326 inhibits cell proliferation as well as oncogenic HH pathway (53). Similarly, Du et al (54) reported that miR-326 inhibits glioma biological behavior and stemness 
through targeting and inhibiting SMO-mediated HH signaling pathway. These findings support the notion that targeting SMO-mediated $\mathrm{HH}$ signaling by specific miRNAs is a promising strategy for MM. In this study, we identified miR-1271 as a novel miRNA that can target SMO and inhibit the $\mathrm{HH}$ signaling pathway in MM suggesting a novel and promising molecular target for MM treatment.

In conclusion, the present study for the first time demonstrated a tumor suppressive role of miR-1271 in MM. We have elucidated that miR-1271 inhibits proliferation and induces apoptosis of MM cells through targeting and inhibition of SMO, leading to the inhibition of HH signaling pathway. Our findings not only improve the understanding of MM pathogenesis, but also provide a potential and promising molecular target for MM therapy development.

\section{References}

1. Hatzimichael E, Dasoula A, Benetatos L, Syed N, Dranitsaris G Crook T and Bourantas K: Study of specific genetic and epigenetic variables in multiple myeloma. Leuk Lymphoma 51: 2270-2274, 2010.

2. Kyle RA and Rajkumar SV: Multiple myeloma. Blood 111: 2962-2972, 2008.

3. Agarwal A and Mahadevan D: Novel targeted therapies and combinations for the treatment of multiple myeloma. Cardiovasc Hematol Disord Drug Targets 13: 2-15, 2013.

4. Siegel RL, Miller KD and Jemal A: Cancer statistics, 2016. CA Cancer J Clin 66: 7-30, 2016.

5. Rossi M, Tagliaferri $\mathrm{P}$ and Tassone P: MicroRNAs in multiple myeloma and related bone disease. Ann Transl Med 3: 334, 2015

6. Bartel DP: MicroRNAs: Genomics, biogenesis, mechanism, and function. Cell 116: 281-297, 2004.

7. Winter J, Jung S, Keller S, Gregory RI and Diederichs S: Many roads to maturity: microRNA biogenesis pathways and their regulation. Nat Cell Biol 11: 228-234, 2009.

8. Mendell JT and Olson EN: MicroRNAs in stress signaling and human disease. Cell 148: 1172-1187, 2012.

9. Liu Z, Zhang G, Yu W, Gao N and Peng J: miR-186 inhibits cell proliferation in multiple myeloma by repressing Jagged1 Biochem Biophys Res Commun 469: 692-697, 2016.

10. Liang B, Yin JJ and Zhan XR: MiR-301a promotes cell proliferation by directly targeting TIMP 2 in multiple myeloma. Int J Clin Exp Pathol 8: 9168-9174, 2015.

11. Lu Y, Wu D, Wang J, Li Y, Chai X and Kang Q: miR-320a regulates cell proliferation and apoptosis in multiple myeloma by targeting pre-B-cell leukemia transcription factor 3 . Biochem Biophys Res Commun 473: 1315-1320, 2016.

12. Saha MN, Abdi J, Yang Y and Chang H: MiRNA-29a as a tumor suppressor mediates PRIMA-1Met-induced anti-myeloma activity by targeting c-Myc. Oncotarget 7: 7149-7160, 2016.

13. Yang Y, Li F, Saha MN, Abdi J, Qiu L and Chang H: miR-137 and miR-197 induce apoptosis and suppress tumorigenicity by targeting MCL-1 in multiple myeloma. Clin Cancer Res 21: 2399-2411, 2015.

14. Zhao JJ, Lin J, Zhu D, Wang X, Brooks D, Chen M, Chu ZB, Takada K, Ciccarelli B, Admin S, et al: miR-30-5p functions as a tumor suppressor and novel therapeutic tool by targeting the oncogenic Wnt/ $\beta$-catenin/BCL9 pathway. Cancer Res 74: 1801-1813, 2014.

15. Zhang Q, Yan W, Bai Y, Xu H, Fu C, Zheng W, Zhu Y and Ma J: Synthetic miR-145 mimic inhibits multiple myeloma cell growth in vitro and in vivo. Oncol Rep 33: 448-456, 2015.

16. Seckinger A, Meißner T, Moreaux J, Benes V, Hillengass J, Castoldi M, Zimmermann J, Ho AD, Jauch A, Goldschmidt H, et al: miRNAs in multiple myeloma - a survival relevant complex regulator of gene expression. Oncotarget 6: 39165-39183, 2015.

17. Sedlaříková L, Bešše L, Novosadová S, Kubaczková V, Radová L, Staník M, Krejčí M, Hájek R and Ševčíková S: MicroRNAs in urine are not biomarkers of multiple myeloma. J Negat Results Biomed 14: 16, 2015.

18. Besse L, Sedlarikova L, Kryukov F, Nekvindova J, Radova L, Slaby O, Kuglik P, Almasi M, Penka M, Krejci M, et al: Circulating serum MicroRNA-130a as a novel putative marker of extramedullary myeloma. PLoS One 10: e0137294, 2015.
19. Ingham PW and McMahon AP: Hedgehog signaling in animal development: Paradigms and principles. Genes Dev 15: 3059-3087, 2001.

20. Hooper JE and Scott MP: Communicating with Hedgehogs. Nat Rev Mol Cell Biol 6: 306-317, 2005.

21. Mann RK and Beachy PA: Novel lipid modifications of secreted protein signals. Annu Rev Biochem 73: 891-923, 2004.

22. Taipale J, Cooper MK, Maiti T and Beachy PA: Patched acts catalytically to suppress the activity of Smoothened. Nature 418: 892-897, 2002.

23. Lum L and Beachy PA: The Hedgehog response network: Sensors, switches, and routers. Science 304: 1755-1759, 2004.

24. Beachy PA, Karhadkar SS and Berman DM: Tissue repair and stem cell renewal in carcinogenesis. Nature 432: 324-331, 2004.

25. Kinzler KW, Bigner SH, Bigner DD, Trent JM, Law ML, O'Brien SJ, Wong AJ and Vogelstein B: Identification of an amplified, highly expressed gene in a human glioma. Science 236: 70-73, 1987.

26. Lee Y, Miller HL, Jensen P, Hernan R, Connelly M, Wetmore C, Zindy F, Roussel MF, Curran T, Gilbertson RJ, et al: A molecular fingerprint for medulloblastoma. Cancer Res 63: 5428-5437, 2003.

27. Katoh Y and Katoh M: Hedgehog target genes: Mechanisms of carcinogenesis induced by aberrant hedgehog signaling activation. Curr Mol Med 9: 873-886, 2009.

28. Suzman DL and Antonarakis ES: Clinical implications of Hedgehog pathway signaling in prostate cancer. Cancers (Basel) 7: 1983-1993, 2015.

29. Huang L, Walter V, Hayes DN and Onaitis M: Hedgehog-GLI signaling inhibition suppresses tumor growth in squamous lung cancer. Clin Cancer Res 20: 1566-1575, 2014.

30. Onishi $\mathrm{H}$ and Katano $\mathrm{M}$ : Hedgehog signaling pathway as a new therapeutic target in pancreatic cancer. World J Gastroenterol 20: 2335-2342, 2014

31. Lindemann RK: Stroma-initiated hedgehog signaling takes center stage in B-cell lymphoma. Cancer Res 68: 961-964, 2008.

32. Tam M, Lin P, Hu P and Lennon PA: Examining Hedgehog pathway genes GLI3, SHH, and PTCH1 and the p53 target GLIPR1/GLIPR1L1/GLIPR1L2 gene cluster using fluorescence in situ hybridization uncovers GLIPR1/GLIPR1L1/GLIPR1L2 deletion in $9 \%$ of patients with multiple myeloma. J Assoc Genet Technol 36: 111-114, 2010.

33. Peacock CD, Wang Q, Gesell GS, Corcoran-Schwartz IM, Jones E, Kim J, Devereux WL, Rhodes JT, Huff CA, Beachy PA, et al: Hedgehog signaling maintains a tumor stem cell compartment in multiple myeloma. Proc Natl Acad Sci USA 104: 4048-4053, 2007.

34. Liu Z, Xu J, He J, Zheng Y, Li H, Lu Y, Qian J, Lin P, Weber DM, Yang J, et al: A critical role of autocrine sonic hedgehog signaling in human $\mathrm{CD} 138^{+}$myeloma cell survival and drug resistance. Blood 124: 2061-2071, 2014.

35. de la Puente P, Muz B, Azab F, Luderer M and Azab AK: Molecularly targeted therapies in multiple myeloma. Leukemia Res Treat 2014: 976567, 2014.

36. Agarwal JR, Wang Q, Tanno T, Rasheed Z, Merchant A, Ghosh N, Borrello I, Huff CA, Parhami F and Matsui W: Activation of liver $\mathrm{X}$ receptors inhibits hedgehog signaling, clonogenic growth, and self-renewal in multiple myeloma. Mol Cancer Ther 13: 1873-1881, 2014.

37. Liu H, Wang H, Liu $X$ and Yu T: miR-1271 inhibits migration, invasion and epithelial-mesenchymal transition by targeting ZEB1 and TWIST1 in pancreatic cancer cells. Biochem Biophys Res Commun 472: 346-352, 2016.

38. Zhou Z, Niu X, Li C, Sheng S and Lu S: Inhibition of the growth of non-small cell lung cancer by miRNA-1271. Am J Transl Res 7: 1917-1924, 2015.

39. Liu X, Ma L, Rao Q, Mao Y, Xin Y, Xu H, Li C and Wang X: MiR-1271 inhibits ovarian cancer growth by targeting cyclin G1. Med Sci Monit 21: 3152-3158, 2015.

40. Xiang XJ, Deng J, Liu YW, Wan LY, Feng M, Chen J and Xiong JP: MiR-1271 inhibits cell proliferation, invasion and EMT in gastric cancer by targeting FOXQ1. Cell Physiol Biochem 36: 1382-1394, 2015.

41. Hönemann D, Chatterjee M, Savino R, Bommert K, Burger R, Gramatzki M, Dörken B and Bargou RC: The IL-6 receptor antagonist SANT-7 overcomes bone marrow stromal cellmediated drug resistance of multiple myeloma cells. Int J Cancer 93: 674-680, 2001. 
42. Rimkus TK, Carpenter RL, Qasem S, Chan M and Lo HW: Targeting the sonic Hedgehog signaling pathway: Review of smoothened and GLI inhibitors. Cancers (Basel) 8: 8, 2016.

43. Chira P, Vareli K, Sainis I, Papandreou C and Briasoulis E: Alterations of MicroRNAs in solid cancers and their prognostic value. Cancers (Basel) 2: 1328-1353, 2010.

44. Maurel M, Jalvy S, Ladeiro Y, Combe C, Vachet L, Sagliocco F, Bioulac-Sage P, Pitard V, Jacquemin-Sablon H, ZucmanRossi J, et al: A functional screening identifies five microRNAs controlling glypican-3: Role of miR-1271 down-regulation in hepatocellular carcinoma. Hepatology 57: 195-204, 2013.

45. Yang M, Shan X, Zhou X, Qiu T, Zhu W, Ding Y, Shu Y and Liu P: miR-1271 regulates cisplatin resistance of human gastric cancer cell lines by targeting IGF1R, IRS1, mTOR, and BCL2. Anticancer Agents Med Chem 14: 884-891, 2014.

46. Kong D, Zhang G, Ma H and Jiang G: miR-1271 inhibits OSCC cell growth and metastasis by targeting ALK. Neoplasma 62: 559-566, 2015

47. Wang Y, Xu L and Jiang L: miR-1271 promotes non-small-cell lung cancer cell proliferation and invasion via targeting HOXA5. Biochem Biophys Res Commun 458: 714-719, 2015.

48. Blotta S, Jakubikova J, Calimeri T, Roccaro AM, Amodio N, Azab AK, Foresta U, Mitsiades CS, Rossi M, Todoerti K, et al: Canonical and noncanonical Hedgehog pathway in the pathogenesis of multiple myeloma. Blood 120: 5002-5013, 2012.
49. Sandhiya S, Melvin G, Kumar SS and Dkhar SA: The dawn of hedgehog inhibitors: Vismodegib. J Pharmacol Pharmacother 4: 4-7, 2013.

50. Huang XH, Chen JS, Wang Q, Chen XL, Wen L, Chen LZ, Bi J, Zhang LJ, Su Q and Zeng WT: miR-338-3p suppresses invasion of liver cancer cell by targeting smoothened. J Pathol 225: 463-472, 2011.

51. Sun K, Deng HJ, Lei ST, Dong JQ and Li GX: miRNA-338-3p suppresses cell growth of human colorectal carcinoma by targeting smoothened. World J Gastroenterol 19: 2197-2207, 2013.

52. Xue Q, Sun K, Deng HJ, Lei ST, Dong JQ and Li GX: MicroRNA338-3p inhibits colorectal carcinoma cell invasion and migration by targeting smoothened. Jpn J Clin Oncol 44: 13-21, 2014.

53. Babashah S, Sadeghizadeh M, Hajifathali A, Tavirani MR Zomorod MS, Ghadiani M and Soleimani M: Targeting of the signal transducer Smo links microRNA-326 to the oncogenic Hedgehog pathway in CD34 ${ }^{+} \mathrm{CML}$ stem/progenitor cells. Int J Cancer 133: 579-589, 2013.

54. Du W, Liu X, Chen L, Dou Z, Lei X, Chang L, Cai J, Cui Y, Yang D, Sun Y, et al: Targeting the SMO oncogene by miR-326 inhibits glioma biological behaviors and stemness. Neuro Oncol 17: 243-253, 2015. 